\title{
Effects of Computer-Aided Decision Support Systems on Appropriate Antibiotic Prescribing by Medical Interns: A Quasi-Experimental Study
}

\author{
Fatemeh RANGRAZ JEDDI ${ }^{\mathrm{a}, \mathrm{b}}$, Mansooreh MOMEN-HERAVI ${ }^{\mathrm{c}, \mathrm{d}}$, \\ Ehsan NABOVATI ${ }^{\mathrm{a}, \mathrm{b}}$, Felix HOLL ${ }^{\mathrm{e}, \mathrm{f}, \mathrm{g}}$, Hossein AKBARI ${ }^{\mathrm{h}, \mathrm{i}}$ and Razieh FARRAHI ${ }^{\mathrm{b}, 1}$ \\ ${ }^{a}$ Health Information Management Research Center, Kashan University of Medical \\ Sciences, Kashan, Iran \\ ${ }^{b}$ Department of Health Information Management \& Technology, School of Allied \\ Health Professions, Kashan University of Medical Sciences, Kashan, Iran \\ ${ }^{c}$ Infectious Diseases Research Center, Kashan University of Medical Sciences, Iran \\ ${ }^{\mathrm{d}}$ Department of Infectious Diseases, School of Medicine, Kashan University of Medical \\ Sciences, Kashan, Iran \\ ${ }^{e}$ Digi Health Institute, Neu-Ulm University of Applied Sciences, Neu-Ulm, Germany \\ ${ }^{f}$ IGHS, University of California, San Francisco, San Francisco, CA, USA \\ ${ }^{g}$ IBE, Ludwig Maximilian University of Munich, Munich, Germany \\ ${ }^{\mathrm{h}}$ Social Determinants of Health (SDH) Research Center, Kashan University of Medical \\ Sciences, Kashan, Iran \\ ${ }^{i}$ Department of Biostatistics and Epidemiology, School of Public Health, Kashan \\ University of Medical Sciences, Kashan, Iran
}

\begin{abstract}
Literature suggests that the adoption of guidelines for antibiotic prescribing has a significant impact on improving prescription practices of physicians; thus, this study aimed to assess the effectiveness of computer-aided decision support systems (CA-DSS) on antibiotic prescribing among medical interns. A prospective before-and-after interventional study was conducted on 40 medical interns. The interns were asked to use the CA-DSS during a one-month internship course at the infectious disease department. The main outcome measure was the knowledge of medical interns regarding the type, name, volume, usual dosages, and administration route of antibiotics prescribed. Paired t-test was applied to assess the change of medical interns' knowledge before and after the study. There was a statistically significant difference between the mean score of interns' medical knowledge before $5.4 \pm 2$ and after $9.1 \pm 2.8$ using the CA-DSS $(\mathrm{p}=0.000)$. CA-DSS as an IT-based training intervention was effective for the knowledge of medical interns to prescribe the right antibiotics for acute respiratory infections.
\end{abstract}

Keywords. Antibiotic, Computer, Prescription, Respiratory tract infection

\footnotetext{
${ }^{1}$ Corresponding author, Razieh Farrahi, Department of Health Information Management \& Technology, School of Allied Health Professions, Kashan University of Medical Sciences, Kashan, Iran; Email: Farrahisr@kaums.ac.ir
} 


\section{Introduction}

Acute respiratory infections are one of the major public health concerns [1] as well as one of the most important reasons for prescribing antibiotics in primary [2] and secondary care [3]. However, most of the prescribed antibiotics are reported as unnecessary and irrational worldwide [4]. Evidence indicates that unnecessary antibiotic prescription might be due to various reasons [5] such as lack of knowledge and failure to apply clinical guidelines reported among the major contributing factors in the unnecessary prescription of antibiotics in healthcare settings [6].

Therefore, developing targeted interventions in terms of training programs, clinical guidelines, and adopting state-of-the-art health information technology (HIT) is acknowledged as effective strategies to improve antibiotics prescription. There is increasing recognition that the education of medical students serves as one of the key approaches to combat the irrational prescription of antibiotics [7].

Given the rapid advancement of HIT and improvement of the digital knowledge and skills of students, instructors, and health professionals at healthcare settings, decision support systems (DSS) hold promises to promote knowledge and practice of rational use of antibiotics among physicians [8]. Adopting DSSs equip with antibiotics guidelines in a knowledge-base of an educational simulator can play a crucial role in improving medical students' knowledge about rational prescription of antibiotics [9]. Since the implementation of these interventions often is accompanied by resources utilization (cost, time, effort, human resources), it is important to investigate to what extent they obtained the desired objectives [10]. Furthermore, researchers have emphasized on background or context in which the intervention is implemented (e.g., hospitals, users, stakeholders, culture), because the impact of any HIT intervention is likely to be affected as much by the background, as the intervention features itself [11]. The purpose of the current study was to examine the effect of using computer-aided decision support systems (CA-DSS) on the appropriate prescription of antibiotics for acute respiratory infections by medical interns.

\section{Method}

We conducted a quasi-experimental study to evaluate the effect of CA-DSS on appropriate antibiotic prescribing by medical interns from March 2021 to July 2021 (5 months) at a general teaching hospital with 510 beds at Kashan University of Medical Sciences (KAUMS) in Iran. Every month, eight medical interns who were spending a one-month internship course at the infectious diseases department participated in the study; forty medical interns participated in the five-month study period. Clinical guidelines and attending physicians' clinical judgment were incorporated as the rules for the knowledge-base of the CA-DSS to perform reasoning and provide educational feedback to medical interns based on targeted scenarios. Sixty scenarios were written by the attending physician via the teacher interface. The correct answers for each scenario including the final diagnosis, signs \& symptoms, diagnostic procedures, and the medication were determined based on guidelines and attending physicians' clinical judgment. The matching method to investigate the right answer was the decision tree via IF-THEN rules. The students were asked to use the CA-DSS while a one-month internship course at the department. After being logged in, a random scenario about acute respiratory infection was displayed to the participants; having read the scenario 
description, the student goes through the four stages of determining the certain diagnosis and signs \& symptoms in favor of diagnosis, diagnostic procedures, appropriate antibiotics, and medication prescription. After responding to each stage, the relevant content of the clinical guideline as well as attending physician recommendations were provided to the medical interns as educational feedback. Finally, the score of each medical intern and the average score of the other participants for the given scenario were also calculated. The main outcome measure was the knowledge of medical interns regarding the diagnosis and treatment of the infection's disease in addition to the appropriate antibiotic's prescription in terms of type, name, volume, usual dosages, and administration route of antibiotics before and after using the CA-DSS. The knowledge scores were measured using two paper-based exams on 14 scenarios, once before the intervention and once after one month applying the CA-DSS. Descriptive statistics, paired t-test and independent t-test were applied to assess the change of medical interns' knowledge score. This study was a part of Ph.D. thesis in the field of health information management that was approved by the Ethics Committee of KAUMS (IR.KAUMS.MEDNT.REC.1398.141) and funded by the deputy of research in Kashan University of Medical Sciences with the grant number (98225).

\section{Results}

These 40 medical interns included 21 men (52.5) and 19 women (47.5), of whom, $17(42.5 \%)$ were sixth-year medical students and 23 (57.5\%) were seventh-year medical students. The CA-DSS was used 369 times by the medical interns to read the scenarios during the intervention period. The average studied scenarios for each medical intern were nine. Antibiotics were correctly selected by the medical interns for 248 scenarios. In 128 (34\%) of the studied scenarios, students revised their answers in compliance with the CA-DSS feedbacks. The interns' knowledge score for antibiotic prescription increased from $5.4 \pm 2$ before the intervention to $9.1 \pm 2.8$ after the CA-DSS intervention $(\mathrm{P}<0.001)$. The results of the independent $\mathrm{t}$-test showed that although the percentage of changes in the score of interns who studied nine or more scenarios $(95.7 \pm 110)$ was higher in compared to interns who studied less than nine scenarios $(85.3 \pm 89.5)$, this difference was not statistically significant $(\mathrm{P}=0.74)$. The results of the independent $\mathrm{t}$-test also showed that although the percentage of changes in the score of sixth-year students $(116 \pm 119)$ was higher in compared to seventh-year students $(69 \pm 74)$, these changes were not statistically significant $(\mathrm{P}=0.14)$.

\section{Discussion}

The CA-DSS as an IT-based educational intervention improved the medical interns' knowledge about the appropriate prescription of antibiotics for acute respiratory infections. The results of similar studies also suggest that DSSs can perform best in the most difficult clinical scenarios and improve physicians' knowledge and practice in antibiotic prescribing [12]. Conducted studies confirmed that training by DSS improves the knowledge of medical students significantly [13, 14]; and overcomes the clinical learning environment challenges in terms of limited access to the well-experienced attending physicians, and evidence-based guidelines to support appropriate antibiotic prescription [15]. Therefore, adopting CA-DSS and educational stimulators would 
improve students learning through providing audit and feedback, facilitating access to clinical guidelines, and supporting evidence-based decision making. To our knowledge, this study represents the first attempt in Iran to develop a computer-aided decision support based on the clinical settings context and the preferences for assisting medical interns' antibiotics prescription. The web-based CDSS supports convenient access to the clinical guidelines at any time and any place. Our study has several limitations: the lack of performing regular pre-test and post-test due to the issues associated with the COVID19 pandemic, in addition, the crowded services following the COVID-19 resulted in the medical interns' tiredness which might have led to the less usage of the system or usage without the required focus. Considering the positive impact of the intervention, it is suggested to design such educational decision aids for other diseases. Also using computer-aided stimulators is recommended as an alternative educational tool for training medical students in clinical environments.

\section{References}

[1] Dagne H, Andualem Z, Dagnew B, Taddese AA. Acute respiratory infection and its associated factors among children under-five years attending pediatric ward at University of Gondar Comprehensive Specialized Hospital, Northwest Ethiopia: institution-based cross-sectional study. BMC pediatrics. 2020;20(1):1-7.

[2] O’Connor R, O'Doherty J, O'Regan A, Dunne C. Antibiotic use for acute respiratory tract infections (ARTI) in primary care; what factors affect prescribing and why is it important? A narrative review. Irish Journal of Medical Science (1971-). 2018;187(4):969-86.

[3] Hussein RA. Patterns of Documenting Patients' Medical Records and Antibiotic Use for Cases with Acute Respiratory Infection in Basrah hospitals. University of Thi-Qar Journal of Medicine. 2018;15(1):1-11.

[4] Machowska A, Stålsby Lundborg C. Drivers of Irrational Use of Antibiotics in Europe. Int J Environ Res Public Health. 2018;16(1):27.

[5] Organization WH. WHO global strategy for containment of antimicrobial resistance. World Health Organization; 2001.

[6] Fleming A, Bradley C, Cullinan S, Byrne S. Antibiotic prescribing in long-term care facilities: a qualitative, multidisciplinary investigation. BMJ open. 2014;4(11): e006442.

[7] Laxminarayan R, Duse A, Wattal C, Zaidi AK, Wertheim HF, Sumpradit N, et al. Antibiotic resistancethe need for global solutions. The Lancet infectious diseases. 2013;13(12):1057-98.

[8] Wasserman S, Potgieter S, Shoul E, Constant D, Stewart A, Mendelson M, et al. South African medical students' perceptions and knowledge about antibiotic resistance and appropriate prescribing: Are we providing adequate training to future prescribers? South African medical journal = Suid-Afrikaanse tydskrif vir geneeskunde. 2017;107(5):405-10.

[9] Willis MH, Frigini LA, Lin J, Wynne DM, Sepulveda KA. Clinical decision support at the point-of-order entry: an education simulation pilot with medical students. Academic radiology. 2016;23(10):1309-18.

[10] Hawe P. Lessons from complex interventions to improve health. Annual review of public health. 2015;36:307-23.

[11] Greenhalgh T, Papoutsi C. Studying complexity in health services research: desperately seeking an overdue paradigm shift. Springer; 2018.

[12] Warner Jr H, Reimer L, Suvinier D, Li L, Nelson M, editors. Modeling empiric antibiotic therapy evaluation of QID. Proceedings of the AMIA Symposium; 1999: American Medical Informatics Association.

[13] Karakuş A, Duran L, Yavuz Y, Altintop L, Çalişkan F. Computer-based simulation training in emergency medicine designed in the light of malpractice cases. BMC Medical Education. 2014;14(1):155.

[14] Leung GM, Johnston JM, Tin KY, Wong IO, Ho LM, Lam WW, et al. Randomised controlled trial of clinical decision support tools to improve learning of evidence-based medicine in medical students. BMJ (Clinical research ed). 2003;327(7423):1090.

[15] Khumrin P, Ryan A, Juddy T, Verspoor K, editors. DrKnow: A diagnostic learning tool with feedback from automated clinical decision support. AMIA Annual Symposium Proceedings; 2018: American Medical Informatics Association. 